\title{
A COMPREHENSIVE STUdy OF DSCP MARKINGS' IMPACT ON VOIP QOS IN HFC NETWORKS
}

\author{
Shaher Daoud and Yanzhen Qu \\ School of Computer Science, Colorado Technical University, Colorado Springs, USA
}

\begin{abstract}
Various factors can have a significant degrading impact on the residential Voice over Internet Protocol (VoIP) phone services' quality. Hybrid fibre-coaxial (HFC) networks typically carry three types of traffic that include voice, data, and video. Unlike data and video, some delays or packet loss can result in a noticeable degraded impact on a VoIP's phone conversation. This paper will analyze and assess VoIP traffic prioritization and its impact on VoIP's quality of service (QoS) based on the concept of differentiated services code point (DSCP) markings. Call testing examines two types of calls. The first set of tests focus on calls that originate from a VoIP network and terminate on a signalling system 7 (SS7) network. The second experiment focuses on calls that originate from SS7 network and terminate on a VoIP network. The research results provide DSCP markings configurations that can improve phone conversations' quality.
\end{abstract}

\section{KEYWORDS}

QoS, VoIP, DSCP Marking, jitter, HFC Network, MOS.

\section{INTRODUCTION}

Globalization changed the overall business environment where it took away the need for companies, suppliers, and customers to be in the same country. Globalization and the Internet help businesses in reducing some of their costs and provide them with some of the means to reach out to more customers. Current advances in communications, including the development of the Internet across the globe, contributed to global business outreach. Some of the large companies like Bank of America and Boeing explored the use of VoIP in their networks to cut costs and to improve their productivity. VoIP is a newly adopted technology; its deployment continues to accelerate [3]. The acronym VoIP refers to voice communication on the Internet using the Internet protocol (IP). Voice signals change into voice packets and get transmitted through the same network that providers use for data communications [4]. VoIP enables voice traffic movement in an IP network. For voice traffic to travel in HFC broadband network, the analog voice signal gets converted into digital signals. Additional hardware such as routers, switches, and servers are needed to transport the initiated voice traffic to its destination. IP broadband networks allow for the combination of voice, data, and video to travel over one single communication link. QoS and security are the primary challenges of VoIP deployment. Packet loss, jitter, and latency continue to be the major elements that impact VoIP's quality [3]. Packet prioritization is an important aspect of VoIP service; it ensures that all packets get to their destination delays and without any packet loss. DSCP markings guarantee packets' prioritization functionality across the different devices in VoIP networks [5]. 
Voice telecommunication started shifting from the traditional public switched telephone network (PSTN) to VoIP. VoIP's low cost is the primary driver of this change. Since VoIP first widespread introduction to the public in February of 1995; VoIP continues to have its voice quality issues. VoIP traffic shares the same links with video and data traffic when it travels through the different network devices including the routers and switches. VoIP's tolerance to packet loss or delay is significantly high where some packet loss or a delay can result in issues such as one-way audio phone calls or callers starting to talk on top of each other [6]. As a result, finding methods that can address VoIP's quality is the primary focus of many scholars and researchers in the telecommunications field. DSCP markings may allow HFC broadband network carriers to prioritize their traffic. If the carriers can to mark the different types of traffic across the various devices in their networks, VoIP's quality might improve. DSCP marking can be of a great benefit to VoIP. This research focuses primarily on assessing the impact of DSCP markings on VoIP traffic.

\section{TOPIC OVERVIEW}

VoIP degraded call quality, when compared with circuit-switched phone calls, became an issue that was not easy to tolerate or ignore. Continuous technology improvements started to have a positive impact on VoIP call quality. Broadband Internet access has expanded worldwide due to continuous developments. Recent technology advancements enabled VoIP service providers to offer better phone service. VoIP service has shifted from providing cheap and low-cost phone calls to real alternative phone service that is similar if not better to the traditional phone services [7]. VoIP is different from PSTN in many ways; differences come from VoIP low-cost, free local, long-distance, and international calls, easy installation, and its scalability. On the other hand, VoIP can have its issues where users might not be able to make calls during power or Internet access outages and its limited support for emergency calls. VoIP travels over the Internet as packets and follows the most efficient route available. On the other hand, PSTN phone calls usually have a dedicated path before the first transmission [8].

For VoIP customers to make and receive calls from traditional PSTN networks, different call setups are involved. Call types include PSTN to VoIP, VoIP to PSTN, PSTN to PSTN, and VoIP to VoIP. Communication networks require three components to accommodate all four-call types; such elements include signalling gateway (SG), a media gateway (MG), and gateway controller (GC). Other VoIP components include IP phones, cable modems, call management system (CMS), VoIP protocols such as session initiation protocol (SIP), and the IP network. IP networks mainly composed of routers and IP switches. Dependent on the VoIP service; some may require additional components such as connectivity to the PSTN network [9]. VoIP, which travels over broadband and wireless services, is becoming a widely used service. VoIP providers were forced to improve VoIP QoS. Some of the common compression/decompression (CODEC) modules that are widely used by VoIP companies include full rate global system for mobile (GSM-FR) with a sampling rate of $13 \mathrm{kbp}$, Global standard 711 (G.711) with a sampling rate of $64 \mathrm{kbps}$, G.723.1 with a sampling frequency of $5.3 \mathrm{kbps}, \&$ G.729A with a sampling rate of $8 \mathrm{kbps}$ [10]. There should be a need for QoS in VoIP calls while at the same time support as many calls as possible [11].

Different elements can that can have an impact on all types of VoIP calls' voice quality. A small delay is one of the acceptable requirements of VoIP applications [12]. The value behind this research is that it guides for the various cable and VoIP providers to improve their residential VoIP phone services. This is important so VoIP providers do not have to build separate networks for each of their services that include VoIP, data, and video. Rather, these providers can utilize the same network equipment $\&$ links to provide their services while maintaining a good quality phone service through voice packets' prioritization. 


\section{Purpose, Problem and Hypothesis}

\subsection{Research Purpose}

This research primarily focuses on studying the quality of VoIP service delivery in an HFC broadband network when mixed with data and video traffic. Dependency on VoIP phone services is on the rise while dependency on the plain old telephone service (POTS) is on the decline. For the period from June 2009 through June of 2012, VoIP services continue to grow and at the same time POTS lines' count continues to decline. VoIP started to dominate traditional POTS lines [13]. By June 2012, there were 90 million switched access lines in service. 45 million lines of that number were for VoIP subscriptions. Between June 2010 and June 2013, VoIP subscription increased 16\% [14]. In broadband HFC networks, VoIP, video, and data share the same transport links including many of the network devices. VoIP sensitivity to packet loss and delay is different from video and data traffic. VoIP traffic cannot tolerate packet loss or delay. The acceptable latency that can be unnoticed should be less than 150 thousandths of seconds (ms) for one way. If latency exceeds $300 \mathrm{~ms}$, then voice quality starts to suffer. In a VoIP conversation with an average delay of $300 \mathrm{~ms}$, both callers begin to talk over each other [15]. DSCP markings may play a critical role in VoIP traffic traveling across a broadband HFC network. This study aids the field of VoIP DSCP markings' research; it uses a quantitative approach in a lab environment. The testing environment takes place in a facility equipped with all the necessary components that HFC broadband network needs to simulate VoIP services offered by cable operators in a production environment. The independent variables of this research included class selector 0 (CS0), CS1, CS3, CS4, and expedited forwarding (EF). The dependent variables included the average mean opinion score (MOS) captured by the Iris tool, calling party's estimated MOS, called party estimated MOS, average jitter, average inter-packet arrival time, latency, and packets lost. This research may benefit the different VoIP carriers to improve the quality of their VoIP traffic offered to their subscribers. Carriers can request and negotiate with other carriers in the telecommunications industry to prioritize their carried VoIP traffic $[1,2]$.

\subsection{Problem Statement}

The primary issue that continues to face VoIP is its QoS. HFC networks carry multiple types of traffic that include voice, data, and video. Some of the elements that affect VoIP quality are packet loss, jitter, latency, and delay. Treating all three types of traffic equally in each element of the broadband HFC network, especially with over-utilized capacity links, can degrade VoIP's QoS $[1,2]$.

\subsection{Hypothesis Statement}

Configuring VoIP traffic with a DSCP marking the value of EF across all the equipment in a broadband HFC network improves VoIP's QoS $[1,2]$.

\subsection{Research Questions}

This research focuses on addressing two primary research question: considering the routers' links carry Internet, video, and VoIP traffic where traffic links are under-utilized. First, will the changes in DSCP markings for the voice traffic have an impact on calls that originate from VoIP networks and terminate in SS7 networks? Second, will the changes in DSCP markings for the voice traffic have an impact on calls that originate from SS7 networks and terminate in VoIP networks? 


\section{RELATED WORKS}

VoIP is called Internet telephony, and it is considered a protocol that allows its users to make telephone calls over the Internet [8]. VoIP's first form was successfully established in 1974 using the Advanced Research Projects Agency Network (ARPANET) that preceded the Internet. It all began in 1966 with two threads. The first was in December of 1966 through Shuzo Saito of Nippon Telegraph and Telephone (NTT) and Fumitada Itakura, who was a doctoral student at Nagoya University. Saito and Itakura published a report at the NTT Electrical Communications Lab; the report provided speech coding's statistical approach. The main idea was to form a model while observing speech data, where the model's description happens and gets created by regression a coefficient or by linear prediction (LP) [16]. The second thread goes back to 1966 at the University of California at Santa Barbara (UCSB). Glen Culler presented his On-Line system that was the first of its kind to provide a Digital Signal Process (DSP) in a classroom environment. This same system allowed signal processing in real-time [16]. The first successful VoIP phone call was made in August of 1974 [17].

Network Voice Protocol (NVP) was tested successfully in real-time using continuously variable slope delta (CVSD) at $16 \mathrm{~kb} / \mathrm{s}$ but with a poor quality of speech. In December of 1974, the first experiment of real-time two-way line predictive coding (LPC) speech occurred and got documented between Culler-Harrison, Inc., and Lincoln Lab. This experiment used $3.5 \mathrm{~kb} / \mathrm{s} \mathrm{[16].}$ The separation of IP from TCP goes back to August of 1977. Cohen, Cerf, and Postel agreed to separate IP from TCP so real-time applications can be allowed. The creation of the User Datagram Protocol (UDP) was the first step that took place. The official date of IP and TCP separation was in January of 1978 [16]. VoIP first breakthrough of its use by the public came in 1996 from a company called VocalTec. The discovery allowed voice transmission over the Internet using IP. Many journalists and researchers believed then that Internet telephony was going to have a tremendous impact on the future of traditional telecommunication sector. The shift to VoIP was expected to come from the high prices of traditional national and international calls. The same calls were free if they were made using VoIP technology that was using Internet service providers' (ISP's) flat rate. VoIP low-cost rates advantage was the primary driver of continuous improvement of VoIP service. VocalTec VoIP calls solution has its limitation; the calls were between personal computers or in other words; the personal computer to personal computer (PC-to-PC). In the middle of the 1990s; other companies such as Deltathree and Net2Phone decided to take this VoIP solution a step further by installing some gateways in the PSTN. The new design enabled telephone-to-PC and PC-to-telephone calls. Quality was not as great when compared to traditional PSTN voice calls. Regardless, during that time; users were experiencing degraded service from their speakerphones and cellular phones. VoIP degraded call quality experience by then was more reasonable and acceptable due to its low-cost when compared to traditional phone calls [7].

The use of multimedia services such as VoIP, video streaming, and video telephony has increased and became a common thing in our daily lives. The transmission of these services rides over IP networks; the size of these networks grew to accommodate the demand. Many of the multimedia services can be sensitive to its allocated bandwidth; decreased bandwidth can translate to degradation of QoS. Some specific high traffic loads can have a negative influence on QoS parameters. The higher the volume of background traffic the higher the percentage of packet loss and jitter can be [18]. 
VoIP and QoS have advanced extremely in the past eight years or so. Research studies on QoS in wireless, mobile, and IP networks increased since. VoIP service is rising and is taking over traditional PSTN. The need for prioritizing voice traffic on IP networks became imminent.

The primary focus of recent research studies focused on VoIP QoS improvement. The implication of recent research studies on VoIP and QoS increased to improve VoIP services and make them affordable, cheaper, and reliable for users' daily needs [19,20,21]. The quality of VoIP traffic can be dependent on five factors: MOS, jitter, latency, network load, and network throughput [22]. There is an imminent need for QoS in VoIP calls while at the same time have the ability to support as many calls as possible [23].

VoIP's degraded call quality, when compared with circuit-switched phone calls, became an issue that was not easy to tolerate or ignore. Since then, many research studies started to focus on improving the quality of VoIP's services. Due to VoIP's nature where it travels across various networks and equipment, many academics \& practitioners continue to study the various aspects of VoIP to improve its service. VoIP's poor QoS through WiFi, UMTS \& WiFi UMTS networks led researchers to examine the MOS and the packets' end-to-end delay to improve VoIP's quality [24]. Some studies turned towards utilizing Machine Learning Quality of Experience (MLQoE) to improve VoIP traffic's quality of Experience. It is left for MLQoE to select the Machine Learning (ML) algorithm that displays the best performance and its parameters automatically given the dataset used as input [25]. There is a recommendation to prioritize Voice traffic to handle packet losses in VoIP services. This can be achieved by providing a mechanism to drop first the least important content, to keep the best VoIP's quality signal for user perception using an Arduino platform [26]. Other researchers investigated the performances of routing protocols Optimized Link State Routing (OLSR) and TRADOC (Training \& Doctrine Command) Operations Research Agency (TORA) in a Mobile Ad hoc Networks (MANETs). This is a group of wireless mobile nodes that form a temporary network without utilizing any centralized access point administration of the mobile networks. The research used the network simulator Optimized Network Engineering Tool (OPNET) 14.5 to analyze and evaluate some QoS metrics like end-to-end delay, Jitter, throughput, and MOS. The OPNET simulation results showed that the OLSR protocol is a good candidate for VoIP application [27].

\section{RESEARCH DESIGN}

This research uses an experimental testing design approach where its quantitative study investigates the impact of changing DSCP markings on VoIP phone calls. The experiments in this study rely on making manual VoIP phone calls. In each of the manual testing scenarios; two testers make and answer the phone calls. Primary elements of the test environment include a Cisco, router and a Nokia 5060 media gateway controller (MGC). The links between the router and the HFC broadband network carry all three types of traffic that include data, video, and VoIP. VoIP traffic marking changes take place at the MGC level. The configurations in the routers rely on the MGCF's VoIP traffic markings. The routers pass VoIP traffic and prioritize it according to its MGCF's DSCP marking. The Iris tool captures the end-to-end call signaling. Iris traces will clearly show the MOS, DSCP markings, and any packet loss or packet drops $[1,2]$.

\subsection{VoIP Phone Calls' Quality}

Differentiated Services (DiffServ) field has eight bits; six of those bits that include zero through five are used for DSCP markings while the sixth and seventh bits are currently unused (CU). Packet prioritization is an important aspect of VoIP services, where it ensures that all packets get 
to their destination without delays or packet loss. DSCP marking follows through packets' prioritization functionality across the different devices in a VoIP network [5]. To ensure testings' consistency; the same data for each of the test calls associated with the two proposed experiments are collected. Each experiment is composed of five scenarios where each scenario is based on making 10 phone calls. The captured data include the average call quality's (CQ) MOS, calling party's MOS, called party's MOS, the average interpacket time in $\mathrm{ms}$, the average jitter in ms, total packets, lost packets, packets' loss percentage, and latency $[1,2]$.

\subsubsection{Manual Process of Phone Calls Testing - VoIP to SS7}

This testing scenario is composed of five independent DSCP marking configuration changes that include class CS0, CS1, CS3, CS4, and EF. The configuration changes take place in the MGC. The testing process starts with logging into the MGC, ensuring there are no active alarms that interfere with the research testing. The process of making 10 VoIP-based calls takes place after each of the DSCP configuration changes [1,2].

\subsubsection{Manual Process of Phone Calls Testing - SS7 to VoIP}

This testing scenario is composed of five independent DSCP marking configuration changes that include CS0, CS1, CS3, CS4, and EF. The configuration changes take place in the MGC. The testing process starts with logging into the MGC, ensuring there are no active alarms that interfere with the research testing. The testing process starts with logging into the MGC, ensuring there are no active alarms that interfere with the research testing. The process of making 10 SS7-based calls takes place after each of the DSCP configuration changes [1,2].

\subsection{Answering the Research Questions}

The experiments are designed in ways that enable the testers to execute each of the tests individually. The experiments' data for each of the test calls are collected and populated in identical tables that are similar to Table 1 . Then, the collected data gets analyzed and used to answer each of the four research questions including the research hypotheses. The analysis is based on the telecom industry and ITU's standards. There is an international recognition for MOS as the preferred metrics that can provide accurate voice quality measurements as observed by the called party. The dependent variables of this research include the average MOS captured by the Iris tool, calling party's estimated MOS, called party estimated MOS, average jitter, average inter-packet arrival time, latency, and packets lost. This research compares the experiment dependent variables' values with the VoIP industry's standards. The MOS standard considers any of the values between 4 and 5 as a clear phone conversation and anything below 4 as poor quality [28]. The acceptable jitter's value is between $0 \mathrm{~ms}$ to $50 \mathrm{~ms}$, and it is unacceptable above $50 \mathrm{~ms}$ [29]. The average interpacket arrival time comes from jitter. If a jitter's value becomes high then it causes the VoIP packets to be out of range, which ends up creating audio issues [30]. VoIP inter-packet arrival time should be between 20 or $30 \mathrm{~ms}$ [31]. The acceptable data loss value falls between $0 \%$ and $1.5 \%$ and unacceptable above this [29]. The maximum tolerable latency value falls between $80 \mathrm{~ms}$ and $120 \mathrm{~ms}$ [32]. We analyze and examine each of the test calls' values thoroughly and separately. 
Table 1. Captured Calls

\begin{tabular}{|l|l|l|l|l|l|l|l|l|}
\hline Call No. & $\begin{array}{l}\text { MOS } \\
\text { Avg } \\
\text { CQ }\end{array}$ & $\begin{array}{l}\text { MOS } \\
\text { Calling } \\
\text { Party }\end{array}$ & $\begin{array}{l}\text { MOS } \\
\text { Called } \\
\text { Party }\end{array}$ & $\begin{array}{l}\text { Avg } \\
\text { Inter- } \\
\text { packet } \\
\text { Time } \\
\text { (ms) }\end{array}$ & $\begin{array}{l}\text { Avg } \\
\text { Jitter } \\
\text { (ms) }\end{array}$ & $\begin{array}{l}\text { Packets } \\
\text { Lost }\end{array}$ & $\begin{array}{l}\text { Packets } \\
\text { Loss } \\
\text { (\%) }\end{array}$ & Latency \\
\hline 1 & & & & & & & & \\
\hline 2 & & & & & & & & \\
\hline 3 & & & & & & & & \\
\hline 4 & & & & & & & & \\
\hline 5 & & & & & & & & \\
\hline 6 & & & & & & & & \\
\hline 7 & & & & & & & & \\
\hline 8 & & & & & & & & \\
\hline 9 & & & & & & & & \\
\hline 10 & & & & & & & & \\
\hline Mean & & & & & & & & \\
\hline Variance & & & & & & & & \\
\hline Deviation & & & & & & & & \\
\hline
\end{tabular}

\section{EXPERIMENTS' DATA}

\subsection{Population and Sample}

A sample represents the general population that shares the same conditions. Quantitative methodology researchers have different sampling method types to choose from. Random sampling is the most appropriate to represent the general population [33]. This experimental research uses a test lab environment owned by a large cable company in the United States. This company uses its lab to test anything related to its VoIP services before their deployment in the field. Lab environment's setup is similar to production environments; the devices used in this research have the same configuration and setup as production environments. Also, there are two testers involved in making the manual test phone calls where one makes a phone call while the other tester answers. Both testers engage in a phone conversation to evaluate the call quality. The caller party and the called party assign a MOS value that is dependent on their evaluation of the quality of the phone conversation. The company that owns the lab provided access and permission to conduct this study. The sample included 10 manual test calls for each of the test cases $[1,2]$.

\subsubsection{Sampling Procedure}

This research is conducted in a lab environment located in Moorestown, New Jersey. The test phone calls are from VoIP to SS7 and from SS7 to VoIP. This research uses samples from both types of call types. The test calls include 10 VoIP to SS7 calls and 10 SS7 to VoIP calls for each of the five DSCP markings. The Cisco routers' configurations do not change any of the markings of the traffic that pass through them. VoIP DSCP configuration changes for each of the test scenarios occur in the MGC. Iris tool is connected directly to the router; its setup enables it to capture all VoIP traffic that passes through the routers. Iris has the capability of storing historical data; the researchers can either capture live calls or go back days or weeks and retrieve the same 
captured calls' data. Each of the call traces has the MOS scores, average interpacket time, average jitter, latency, and any dropped packets. The VoIP calls' samples have the same originating source, the same terminating source, and use the same transport path. This setup avoids bias throughout this research [1,2].

\subsubsection{Instrumentations}

Researchers and engineers are in need of specific instruments they can use to test the different systems or design studies. The need for such tools is related to the need to provide some key measurements that can be hard to get without such instrumentation devices [35]. Iris is one instrument that is being used in this study to capture the end-to-end call traces. Iris is connected to the routers and is set up to capture each and every VoIP calls that pass through them. Iris tool is being used throughout the testing process to capture an end-to-end call signalling messages. Iris uses VoIP phone calls' IDs for each call that is made to correlate each of the test calls' messages. Iris has the capability to provide the network's equipment that can potentially drop any of the packets in each of the test calls. Iris is one of the commonly available instruments that are capable of providing captured measurements needed for this research $[1,2]$.

\subsection{Data Collection}

Data collection involves multiple processes. It covers gaining permission to the testing site. Data collection utilizes a suitable sampling strategy. The sampling plan design was composed to generate 10 VoIP to SS7 calls and 10 SS7 to VoIP calls for each of the tests. Data collection recommendation is to develop the means to record the tests and the data associated with them. This research utilized Iris tool as a digital form for recording the data. Data collection requires the means to store the data. The researcher relied on the Iris tool to store the data and created another two different storage devices like a primary and backup. The primary backup was on the researcher's laptop, and the secondary was on a flash drive [35]. Data collection carries its importance to this research. The Iris tool had the ability and capability of capturing the call traces associated with this research. Data collection tools in experiments are used to support research evaluation [36]. Data collection importance was needed to perform the measurements of this research. There was a need for a collection server to collect the data and measurements for each of the test cases associated with this research. Iris' second usage in this research was as a collection server. Iris stored all the tests related to this research including their measurements. The router had two probe links configured to capture all VoIP traffic signalling and messages including RTP. Both probe links got connected to Iris. Each call had multiple messages between the different network systems. Iris used the tested telephone number and the time to retrieve all messages, signalling, and RTP to provide a trace that had all messages associated with a particular call. Iris trace for any given call was capable of providing end-to-end messages for each of the calls that passed through the probe links. It was possible to look at each of the messages within the trace and validate DSCP marking and packet drops $[1,2]$.

\section{EXPERIMENTS' RESULTS AND ANALYSIS}

This section covers two test scenarios. The first scenario will be based on manually making phone calls from a VoIP network and terminate on the SS7 network. The second scenario will be based on manually making phone calls from the SS7 network and terminate on a VoIP network. Each experiment will be composed of five tests where each of the tests will focus on one DSCP configurations that include CS0, CS1, CS3, CS4, and EF [1, 2]. 


\subsection{VoIP to SS7 Experiments}

This section focuses on VoIP to SS7 test scenarios and their related data.

\subsubsection{VoIP to SS7 Test Calls with a DSCP Marking Value of CSO}

10 random calls are made. The lowest MOS value of the 10 calls shows a value of 4.380 and the highest value is 4.400 . The MOS mean value is 4.396; the variance is 0 , and its highest standard deviation's value is 0.007 . The lowest average inter-packet arrival time is $20.030 \mathrm{~ms}$, while the highest value is $20.400 \mathrm{~ms}$. The mean is $20.056 \mathrm{~ms}$, its variance is 0.015 , and its standard deviation is 0.124 . The lowest jitter's value is $0.01 \mathrm{~ms}$ while the highest value is $5.30 \mathrm{~ms}$. The mean is $1.33 \mathrm{~ms}$, its variance is 3.90 , and its standard deviation is 1.97 . The highest packet loss is $0.27 \%$, and the highest latency value is $54 \mathrm{~ms}$. Examining the values of each of the dependent variables under Table 2 indicates that each of the values was within specifications which lead to good and clear phone conversations between both parties [1,2].

Table 2. VoIP to SS7 test calls with a DSCP marking value of CS0

\begin{tabular}{|l|l|l|l|l|l|l|l|l|}
\hline Call & $\begin{array}{l}\text { MOS } \\
\text { Avg } \\
\text { CQ }\end{array}$ & $\begin{array}{l}\text { MOS } \\
\text { Calling } \\
\text { Party }\end{array}$ & $\begin{array}{l}\text { MOS } \\
\text { Called } \\
\text { Party }\end{array}$ & $\begin{array}{l}\text { Avg } \\
\text { Inter- } \\
\text { packet } \\
\text { Time } \\
(\mathbf{m s})\end{array}$ & $\begin{array}{l}\text { Avg } \\
\text { Jitter } \\
(\mathbf{m s})\end{array}$ & $\begin{array}{l}\text { Packets } \\
\text { Lost }\end{array}$ & $\begin{array}{l}\text { Packets } \\
\text { Loss } \\
(\%)\end{array}$ & Latency \\
\hline Lowest & 4.380 & 4.400 & 4.400 & 20.030 & 0.01 & 0 & 0.00 & 0 \\
\hline Highest & 4.400 & 4.400 & 4.400 & 20.400 & 5.30 & 8 & 0.27 & 54 \\
\hline Mean & 4.396 & 4.400 & 4.400 & 20.056 & 1.33 & 2.4 & - & 23.4 \\
\hline Var & 0.000 & 0.000 & 0.000 & 00.015 & 3.90 & 10.5 & - & 456 \\
\hline Dev & 0.007 & 0.000 & 0.000 & 00.124 & 1.97 & 3.2 & - & 21.4 \\
\hline
\end{tabular}

\subsubsection{VoIP to SS7 test calls with a DSCP marking value of CS1}

All 10 calls have the same MOS value of 4.400. The MOS mean value is 4.400 , the variance is 0 , and its standard deviation's value is 0 . The lowest average inter-packet arrival time is $20.000 \mathrm{~ms}$ while the highest is $20.070 \mathrm{~ms}$. The mean is $20.011 \mathrm{~ms}$, its variance is 0 , and its standard deviation is 0.022 . The lowest jitter's value is $0.02 \mathrm{~ms}$ while the highest is $7.83 \mathrm{~ms}$. The mean is $3.77 \mathrm{~ms}$, its variance is 12.10 , and its standard deviation is 3.48 . Packet loss is $0 \%$, and the highest latency value is $20 \mathrm{~ms}$. Examining the values of each of the dependent variables under Table 3 indicates that each of the values was within specifications that lead to good and clear phone conversations between both parties [1,2]. 
Table 3. VoIP to SS7 test calls with a DSCP marking value of CS1

\begin{tabular}{|l|l|l|l|l|l|l|l|l|}
\hline Call & $\begin{array}{l}\text { MOS } \\
\text { Avg } \\
\text { CQ }\end{array}$ & $\begin{array}{l}\text { MOS } \\
\text { Calling } \\
\text { Party }\end{array}$ & $\begin{array}{l}\text { MOS } \\
\text { Called } \\
\text { Party }\end{array}$ & $\begin{array}{l}\text { Avg } \\
\text { Inter- } \\
\text { packet } \\
\text { Time } \\
(\mathbf{m s})\end{array}$ & $\begin{array}{l}\text { Avg } \\
\text { Jitter } \\
(\mathbf{m s})\end{array}$ & $\begin{array}{l}\text { Packets } \\
\text { Lost }\end{array}$ & $\begin{array}{l}\text { Packets } \\
\text { Loss } \\
(\%)\end{array}$ & Latency \\
\hline Lowest & 4.400 & 4.400 & 4.400 & 20.000 & 0.02 & 0 & 0.00 & 8 \\
\hline Highest & 4.400 & 4.400 & 4.400 & 20.070 & 7.83 & 0 & 0.00 & 20 \\
\hline Mean & 4.400 & 4.400 & 4.400 & 20.011 & 3.77 & 0.00 & - & 12.5 \\
\hline Var & 0.000 & 0.000 & 0.000 & 00.000 & 12.1 & 0.00 & - & 17.4 \\
\hline Dev & 0.000 & 0.000 & 0.000 & 00.022 & 3.48 & 0.00 & - & 4.17 \\
\hline
\end{tabular}

\subsubsection{VoIP to SS7 test calls with a DSCP marking value of CS3}

All 10 calls have the same MOS value of 4.400. The MOS mean value is 4.400 , the variance is 0 , and its standard deviation's value is 0 . The lowest average inter-packet arrival time is $20.000 \mathrm{~ms}$ while the highest is $20.070 \mathrm{~ms}$. The mean is $20.011 \mathrm{~ms}$, its variance is 0 , and its standard deviation is 0.022 . The lowest jitter's value is $0.02 \mathrm{~ms}$ while the highest is $7.83 \mathrm{~ms}$. The mean is $3.77 \mathrm{~ms}$, its variance is 12.10 , and its standard deviation is 3.48 . Packet loss is $0 \%$, and the highest latency value is $20 \mathrm{~ms}$. Examining the values of each of the dependent variables under Table 4 indicate that each of the values was within specifications that lead to good and clear phone conversations between both parties $[1,2]$.

Table 4. VoIP to SS7 test calls with a DSCP marking value of CS3

\begin{tabular}{|l|l|l|l|l|l|l|l|l|}
\hline Call & $\begin{array}{l}\text { MOS } \\
\text { Avg } \\
\text { CQ }\end{array}$ & $\begin{array}{l}\text { MOS } \\
\text { Calling } \\
\text { Party }\end{array}$ & $\begin{array}{l}\text { MOS } \\
\text { Called } \\
\text { Party }\end{array}$ & $\begin{array}{l}\text { Avg } \\
\text { Inter- } \\
\text { packet } \\
\text { Time } \\
(\mathbf{m s})\end{array}$ & $\begin{array}{l}\text { Avg } \\
\text { Jitter } \\
(\mathbf{m s})\end{array}$ & $\begin{array}{l}\text { Packets } \\
\text { Lost }\end{array}$ & $\begin{array}{l}\text { Packets } \\
\text { Loss } \\
(\%)\end{array}$ & Latency \\
\hline Lowest & 4.400 & 4.400 & 4.400 & 20.000 & 0.01 & 0 & 0.00 & 0 \\
\hline Highest & 4.400 & 4.400 & 4.400 & 20.010 & 4.01 & 0 & 0.00 & 8 \\
\hline Mean & 4.400 & 4.400 & 4.400 & 20.001 & 1.11 & 0.00 & - & 0.8 \\
\hline Var & 0.000 & 0.000 & 0.000 & 00.000 & 1.86 & 0.00 & - & 6.4 \\
\hline Dev & 0.000 & 0.000 & 0.000 & 00.003 & 1.37 & 0.00 & - & 2.5 \\
\hline
\end{tabular}

\subsubsection{VoIP to SS7 test calls with a DSCP marking value of CS4}

All 10 calls have the same MOS value of 4.400. The MOS mean value is 4.400 , the variance is 0 , and its standard deviation's value is 0 . The lowest average inter-packet arrival time is $20.000 \mathrm{~ms}$ while the highest is $20.010 \mathrm{~ms}$. The mean is $20.001 \mathrm{~ms}$, its variance is 0 , and its standard deviation is 0.003 . The lowest jitter's value is $0.01 \mathrm{~ms}$ while the highest is $0.02 \mathrm{~ms}$. The mean is $0.02 \mathrm{~ms}$, its variance is 0 , and its standard deviation is 0.01 . Packet loss is $0 \%$, and there is no indication of any latency. Examining the values of each of the dependent variables under Table 5 indicate that each of the values was within specifications that lead to good and clear phone calls between both parties [1, 2]. 
Table 5. VoIP to SS7 test calls with a DSCP marking value of CS4

\begin{tabular}{|l|l|l|l|l|l|l|l|l|}
\hline Call & $\begin{array}{l}\text { MOS } \\
\text { Avg } \\
\text { CQ }\end{array}$ & $\begin{array}{l}\text { MOS } \\
\text { Calling } \\
\text { Party }\end{array}$ & $\begin{array}{l}\text { MOS } \\
\text { Called } \\
\text { Party }\end{array}$ & $\begin{array}{l}\text { Avg } \\
\text { Inter- } \\
\text { packet } \\
\text { Time } \\
(\mathbf{m s})\end{array}$ & $\begin{array}{l}\text { Avg } \\
\text { Jitter } \\
(\mathbf{m s})\end{array}$ & $\begin{array}{l}\text { Packets } \\
\text { Lost }\end{array}$ & $\begin{array}{l}\text { Packets } \\
\text { Loss } \\
(\%)\end{array}$ & Latency \\
\hline Lowest & 4.400 & 4.400 & 4.400 & 20.000 & 0.01 & 0 & 0.00 & 0 \\
\hline Highest & 4.400 & 4.400 & 4.400 & 20.010 & 0.02 & 0 & 0.00 & 0 \\
\hline Mean & 4.400 & 4.400 & 4.400 & 20.001 & 0.02 & 0.00 & - & 0.0 \\
\hline Var & 0.000 & 0.000 & 0.000 & 00.000 & 0.00 & 0.00 & - & 0.0 \\
\hline Dev & 0.000 & 0.000 & 0.000 & 00.003 & 0.01 & 0.00 & - & 0.0 \\
\hline
\end{tabular}

\subsubsection{VoIP to SS7 test calls with a DSCP marking value of EF}

All 10 calls have the same MOS value of 4.400. The MOS mean value is 4.400 , the variance is 0 , and its standard deviation's value is 0 . The average interpacket arrival time for each of the 10 calls is $20.000 \mathrm{~ms}$. The mean is $20.000 \mathrm{~ms}$, its variance is 0 , and its standard deviation is 0 . The highest jitter's value is $0.02 \mathrm{~ms}$. The mean is $0.15 \mathrm{~ms}$, its variance is 0 , and its standard deviation is 0.01 . Packet loss is $0 \%$, and there is no indication of any latency. Examining the values of each of the dependent variables under Table 6 indicate that each of the values was within specifications that lead to good and clear phone calls between both parties [1, 2].

Table 6. VoIP to SS7 test calls with a DSCP marking value of EF

\begin{tabular}{|l|l|l|l|l|l|l|l|l|}
\hline Call & $\begin{array}{l}\text { MOS } \\
\text { Avg } \\
\text { CQ }\end{array}$ & $\begin{array}{l}\text { MOS } \\
\text { Calling } \\
\text { Party }\end{array}$ & $\begin{array}{l}\text { MOS } \\
\text { Called } \\
\text { Party }\end{array}$ & $\begin{array}{l}\text { Avg } \\
\text { Inter- } \\
\text { packet } \\
\text { Time } \\
(\mathbf{m s})\end{array}$ & $\begin{array}{l}\text { Avg } \\
\text { Jitter } \\
(\mathbf{m s})\end{array}$ & $\begin{array}{l}\text { Packets } \\
\text { Lost }\end{array}$ & $\begin{array}{l}\text { Packets } \\
\text { Loss } \\
(\%)\end{array}$ & Latency \\
\hline Lowest & 4.400 & 4.400 & 4.400 & 20.000 & 0.00 & 0 & 0.00 & 0 \\
\hline Highest & 4.400 & 4.400 & 4.400 & 20.000 & 0.02 & 0 & 0.00 & 0 \\
\hline Mean & 4.400 & 4.400 & 4.400 & 20.000 & 0.15 & 0.00 & - & 0.0 \\
\hline Var & 0.000 & 0.000 & 0.000 & 00.000 & 0.00 & 0.00 & - & 0.0 \\
\hline Dev & 0.000 & 0.000 & 0.000 & 00.000 & 0.01 & 0.00 & - & 0.0 \\
\hline
\end{tabular}

\subsection{SS7 to VoIP Experiments}

This section focuses on SS7 to VoIP test scenarios and their related data.

\subsubsection{SS7 to VoIP test calls with a DSCP marking value of CS0}

The lowest MOS value of the 10 calls shows a value of 4.360 and the highest value is 4.400 . The MOS mean value is 4.392; the variance is 0 , and its highest standard deviation's value is 0.013 . The lowest average inter-packet arrival time is $20.000 \mathrm{~ms}$ while the highest is $20.400 \mathrm{~ms}$. The mean is $20.055 \mathrm{~ms}$, its variance is 0.016 , and its standard deviation is 0.125 . The lowest jitter's value is $0.01 \mathrm{~ms}$ while the highest is $5.47 \mathrm{~ms}$. The mean is $0.98 \mathrm{~ms}$, its variance is 3.78 , and its standard deviation is 1.94 . The highest packet loss is $0.49 \%$, and the highest latency value is 54 ms. Examining the values of each of the dependent variables under Table 7 indicate that each of the values was within specifications that lead to good and clear phone conversations between both parties [1,2]. 
Table 7. SS7 to VoIP test calls with a DSCP marking value of CS0

\begin{tabular}{|l|l|l|l|l|l|l|l|l|}
\hline Call & $\begin{array}{l}\text { MOS } \\
\text { Avg } \\
\text { CQ }\end{array}$ & $\begin{array}{l}\text { MOS } \\
\text { Calling } \\
\text { Party }\end{array}$ & $\begin{array}{l}\text { MOS } \\
\text { Called } \\
\text { Party }\end{array}$ & $\begin{array}{l}\text { Avg } \\
\text { Inter- } \\
\text { packet } \\
\text { Time } \\
(\mathbf{m s})\end{array}$ & $\begin{array}{l}\text { Avg } \\
\text { Jitter } \\
(\mathbf{m s})\end{array}$ & $\begin{array}{l}\text { Packets } \\
\text { Lost }\end{array}$ & $\begin{array}{l}\text { Packets } \\
\text { Loss } \\
(\%)\end{array}$ & Latency \\
\hline Lowest & 4.360 & 4.400 & 4.400 & 20.000 & 0.01 & 0 & 0.00 & 0 \\
\hline Highest & 4.400 & 4.400 & 4.400 & 20.400 & 5.47 & 6 & 0.49 & 54 \\
\hline Mean & 4.392 & 4.400 & 4.400 & 20.055 & 0.98 & 1.40 & - & 22.9 \\
\hline Var & 0.000 & 0.000 & 0.000 & 00.016 & 3.78 & 5.38 & - & 413 \\
\hline Dev & 0.013 & 0.000 & 0.000 & 00.125 & 1.94 & 2.32 & - & 20.03 \\
\hline
\end{tabular}

\subsubsection{SS7 to VoIP test calls with a DSCP marking value of CS1}

All 10 calls had the same MOS value of 4.400. The MOS mean value is 4.400 , the variance is 0 , and its standard deviation's value is 0 . The lowest average inter-packet arrival time is $20.000 \mathrm{~ms}$ while the highest is $20.120 \mathrm{~ms}$. The mean is $20.024 \mathrm{~ms}$, its variance is 0.002 , and its standard deviation is 0.042 . The lowest jitter's value is $0.01 \mathrm{~ms}$ while the highest is $6.69 \mathrm{~ms}$. The mean is $2.30 \mathrm{~ms}$, its variance is 6.98 , and its standard deviation is 2.64 . Packet loss is $0 \%$, and the highest latency value is $19 \mathrm{~ms}$. Examining the values of each of the dependent variables under Table 8 indicate that each of the values was within specifications that lead to good and clear phone conversations between both parties $[1,2]$.

Table 8. SS7 to VoIP Test Calls with a DSCP Marking Value of CS1

\begin{tabular}{|l|l|l|l|l|l|l|l|l|}
\hline Call & $\begin{array}{l}\text { MOS } \\
\text { Avg } \\
\text { CQ }\end{array}$ & $\begin{array}{l}\text { MOS } \\
\text { Calling } \\
\text { Party }\end{array}$ & $\begin{array}{l}\text { MOS } \\
\text { Called } \\
\text { Party }\end{array}$ & $\begin{array}{l}\text { Avg } \\
\text { Inter- } \\
\text { packet } \\
\text { Time } \\
(\mathbf{m s})\end{array}$ & $\begin{array}{l}\text { Avg } \\
\text { Jitter } \\
(\mathbf{m s})\end{array}$ & $\begin{array}{l}\text { Packets } \\
\text { Lost }\end{array}$ & $\begin{array}{l}\text { Packets } \\
\text { Loss } \\
(\%)\end{array}$ & Latency \\
\hline Lowest & 4.400 & 4.400 & 4.400 & 20.000 & 0.01 & 0 & 0.00 & 8 \\
\hline Highest & 4.400 & 4.400 & 4.400 & 20.120 & 6.69 & 0 & 0.00 & 19 \\
\hline Mean & 4.400 & 4.400 & 4.400 & 20.024 & 2.30 & 0.00 & - & 11.3 \\
\hline Var & 0.000 & 0.000 & 0.000 & 00.002 & 6.98 & 0.00 & - & 11.8 \\
\hline Dev & 0.000 & 0.000 & 0.000 & 00.042 & 2.64 & 0.00 & - & 3.43 \\
\hline
\end{tabular}

\subsubsection{SS7 to VoIP test calls with a DSCP marking value of CS3}

All 10 calls have the same MOS value of 4.400. The MOS mean value is 4.400 , the variance is 0 , and its standard deviation's value is 0 . The lowest average inter-packet arrival time is $19.990 \mathrm{~ms}$ while the highest is $20.000 \mathrm{~ms}$. The mean is $19.999 \mathrm{~ms}$, its variance is 0 , and its standard deviation is 0.003 . The lowest jitter's value is $0.01 \mathrm{~ms}$ while the highest is $1.10 \mathrm{~ms}$. The mean is $0.20 \mathrm{~ms}$, its variance is 0.10 , and its standard deviation is 0.32 . Packet loss is $0 \%$, and the highest latency value is $8 \mathrm{~ms}$. Examining the values of each of the dependent variables under Table 9 indicate that each of the values was within specifications that lead to good and clear phone calls between both parties $[1,2]$. 
Table 9. SS7 to VoIP Test Calls with a DSCP Marking Value of CS3

\begin{tabular}{|l|l|l|l|l|l|l|l|l|}
\hline Call & $\begin{array}{l}\text { MOS } \\
\text { Avg } \\
\text { CQ }\end{array}$ & $\begin{array}{l}\text { MOS } \\
\text { Calling } \\
\text { Party }\end{array}$ & $\begin{array}{l}\text { MOS } \\
\text { Called } \\
\text { Party }\end{array}$ & $\begin{array}{l}\text { Avg } \\
\text { Inter- } \\
\text { packet } \\
\text { Time } \\
(\mathbf{m s})\end{array}$ & $\begin{array}{l}\text { Avg } \\
\text { Jitter } \\
(\mathbf{m s})\end{array}$ & $\begin{array}{l}\text { Packets } \\
\text { Lost }\end{array}$ & $\begin{array}{l}\text { Packets } \\
\text { Loss } \\
(\%)\end{array}$ & Latency \\
\hline Lowest & 4.400 & 4.400 & 4.400 & 19.990 & 0.01 & 0 & 0.00 & 0 \\
\hline Highest & 4.400 & 4.400 & 4.400 & 20.000 & 1.10 & 0 & 0.00 & 8 \\
\hline Mean & 4.400 & 4.400 & 4.400 & 19.999 & 0.20 & 0.00 & - & 0.8 \\
\hline Var & 0.000 & 0.000 & 0.000 & 00.000 & 0.10 & 0.00 & - & 6.40 \\
\hline Dev & 0.000 & 0.000 & 0.000 & 00.003 & 0.32 & 0.00 & - & 2.53 \\
\hline
\end{tabular}

\subsubsection{SS7 to VoIP test calls with a DSCP marking value of CS4}

All 10 calls have the same MOS value of 4.400. The MOS mean value is 4.400 , the variance is 0 , and its standard deviation's value is 0 . The average inter-packet arrival time for each of the test calls is $20.000 \mathrm{~ms}$. The mean is $20.000 \mathrm{~ms}$, its variance is 0 , and its standard deviation is 0 . The lowest jitter's value is $0.01 \mathrm{~ms}$ while the highest is $0.03 \mathrm{~ms}$. The mean is $0.02 \mathrm{~ms}$, its variance is 0 , and its standard deviation is 0.01 . Packet loss is $0 \%$, and there is no indication of any latency. Examining the values of each of the dependent variables under Table 10 indicate that each of the values was within specifications that lead to good and clear phone calls between both parties [1, 2].

Table 10. SS7 to VoIP test calls with a DSCP marking value of CS4

\begin{tabular}{|l|l|l|l|l|l|l|l|l|}
\hline Call & $\begin{array}{l}\text { MOS } \\
\text { Avg } \\
\text { CQ }\end{array}$ & $\begin{array}{l}\text { MOS } \\
\text { Calling } \\
\text { Party }\end{array}$ & $\begin{array}{l}\text { MOS } \\
\text { Called } \\
\text { Party }\end{array}$ & $\begin{array}{l}\text { Avg } \\
\text { Inter- } \\
\text { packet } \\
\text { Time } \\
(\mathbf{m s})\end{array}$ & $\begin{array}{l}\text { Avg } \\
\text { Jitter } \\
(\mathbf{m s})\end{array}$ & $\begin{array}{l}\text { Packets } \\
\text { Lost }\end{array}$ & $\begin{array}{l}\text { Packets } \\
\text { Loss } \\
(\%)\end{array}$ & Latency \\
\hline Lowest & 4.400 & 4.400 & 4.400 & 20.000 & 0.01 & 0 & 0.00 & 0 \\
\hline Highest & 4.400 & 4.400 & 4.400 & 20.000 & 0.03 & 0 & 0.00 & 0 \\
\hline Mean & 4.400 & 4.400 & 4.400 & 20.000 & 0.02 & 0.00 & - & 0.00 \\
\hline Var & 0.000 & 0.000 & 0.000 & 00.000 & 0.00 & 0.00 & - & 0.00 \\
\hline Dev & 0.000 & 0.000 & 0.000 & 00.003 & 0.01 & 0.00 & - & 0.00 \\
\hline
\end{tabular}

\subsubsection{SS7 to VoIP test calls with a DSCP marking value of EF}

All 10 calls have the same MOS value of 4.400. The MOS mean value is 4.400 , the variance is 0 , and its standard deviation's value is 0 . The average inter-packet arrival time for each of the test calls is $20.000 \mathrm{~ms}$. The mean is $20.000 \mathrm{~ms}$, its variance is 0 , and its standard deviation is 0 . The lowest jitter's value is $0.01 \mathrm{~ms}$ while the highest jitter's value is $0.02 \mathrm{~ms}$. The mean is $0.02 \mathrm{~ms}$, its variance is 0 , and its standard deviation is 0 . Packet loss is $0 \%$, and there is no indication of any latency. Examining the values of each of the dependent variables under Table 11 indicate that each of the values was within specifications that lead to good and clear phone calls between both parties $[1,2]$. 
Table 11. SS7 to VoIP Test Calls with a DSCP Marking Value of EF

\begin{tabular}{|l|l|l|l|l|l|l|l|l|}
\hline Call & $\begin{array}{l}\text { MOS } \\
\text { Avg } \\
\text { CQ }\end{array}$ & $\begin{array}{l}\text { MOS } \\
\text { Calling } \\
\text { Party }\end{array}$ & $\begin{array}{l}\text { MOS } \\
\text { Called } \\
\text { Party }\end{array}$ & $\begin{array}{l}\text { Avg } \\
\text { Inter- } \\
\text { packet } \\
\text { Time } \\
(\mathbf{m s})\end{array}$ & $\begin{array}{l}\text { Avg } \\
\text { Jitter } \\
(\mathbf{m s})\end{array}$ & $\begin{array}{l}\text { Packets } \\
\text { Lost }\end{array}$ & $\begin{array}{l}\text { Packets } \\
\text { Loss } \\
(\%)\end{array}$ & Latency \\
\hline Lowest & 4.400 & 4.400 & 4.400 & 20.000 & 0.01 & 0 & 0.00 & 0 \\
\hline Highest & 4.400 & 4.400 & 4.400 & 20.000 & 0.02 & 0 & 0.00 & 0 \\
\hline Mean & 4.400 & 4.400 & 4.400 & 20.000 & 0.02 & 0.00 & - & 0.00 \\
\hline Var & 0.000 & 0.000 & 0.000 & 00.000 & 0.00 & 0.00 & - & 0.00 \\
\hline Dev & 0.000 & 0.000 & 0.000 & 00.000 & 0.00 & 0.00 & - & 0.00 \\
\hline
\end{tabular}

\subsection{Calls' Data Analysis}

Figure 1 illustrates the MOS' mean values for each of the 10 test cases. All the test cases have a good call quality where none of the MOS values fell below the 4.360 scores. The lowest mean value for MOS is 4.392 which belong to the CS0 marking of the SS7 based manual test calls. This value refers to a good quality VoIP phone conversation. According to VoIP standards; any MOS value between 4 and 5 indicates a good quality phone conversation [1, 2].

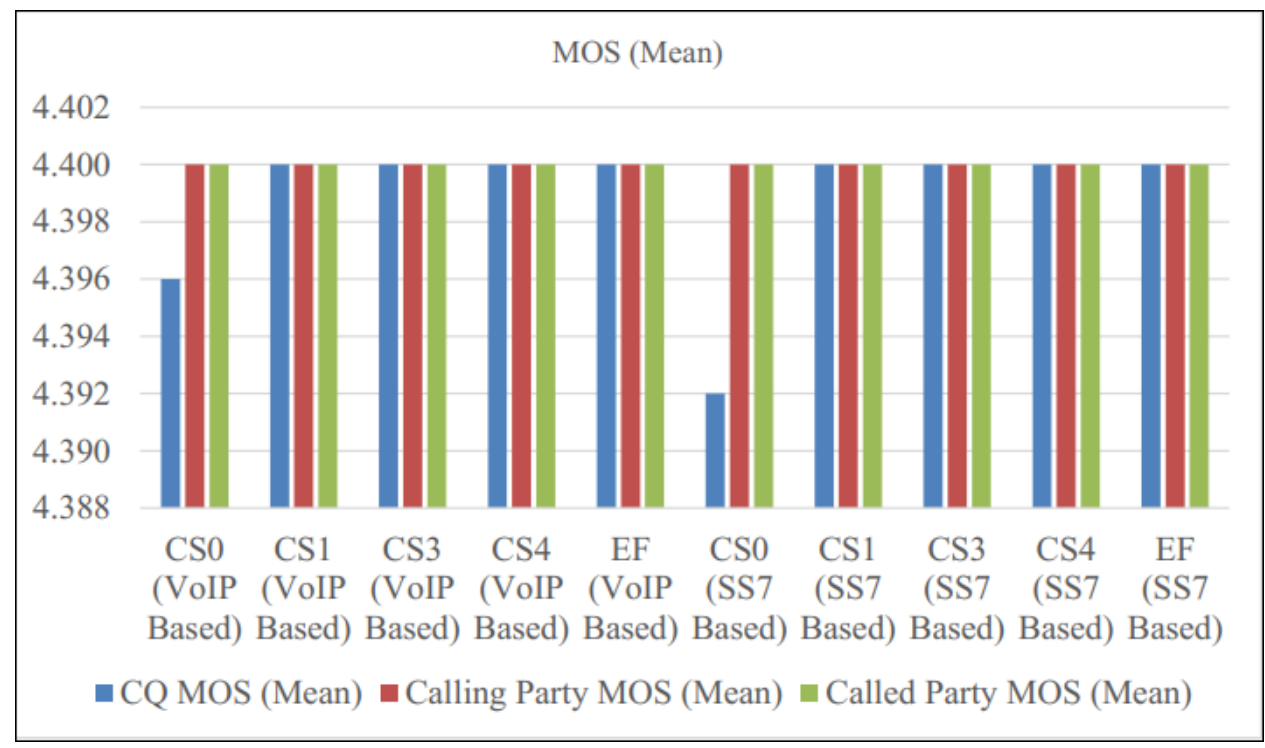

Figure 1. MOS mean

Figure 2 illustrates the average interpacket arrival time's mean values for each of the 10 test cases. All the test cases have a steady and regular interval inter-packet arrival that fell between $19.990 \mathrm{~ms}$ and $20.400 \mathrm{~ms}$. The mean value for the interpacket arrival time associated with each of the 10 test cases is $20 \mathrm{~ms}$. This value goes hand-in-hand with the VoIP standards of good interpacket arrival time of $20 \mathrm{~ms}[1,2]$. 
International Journal of Computer Networks \& Communications (IJCNC) Vol.11, No.5, September 2019

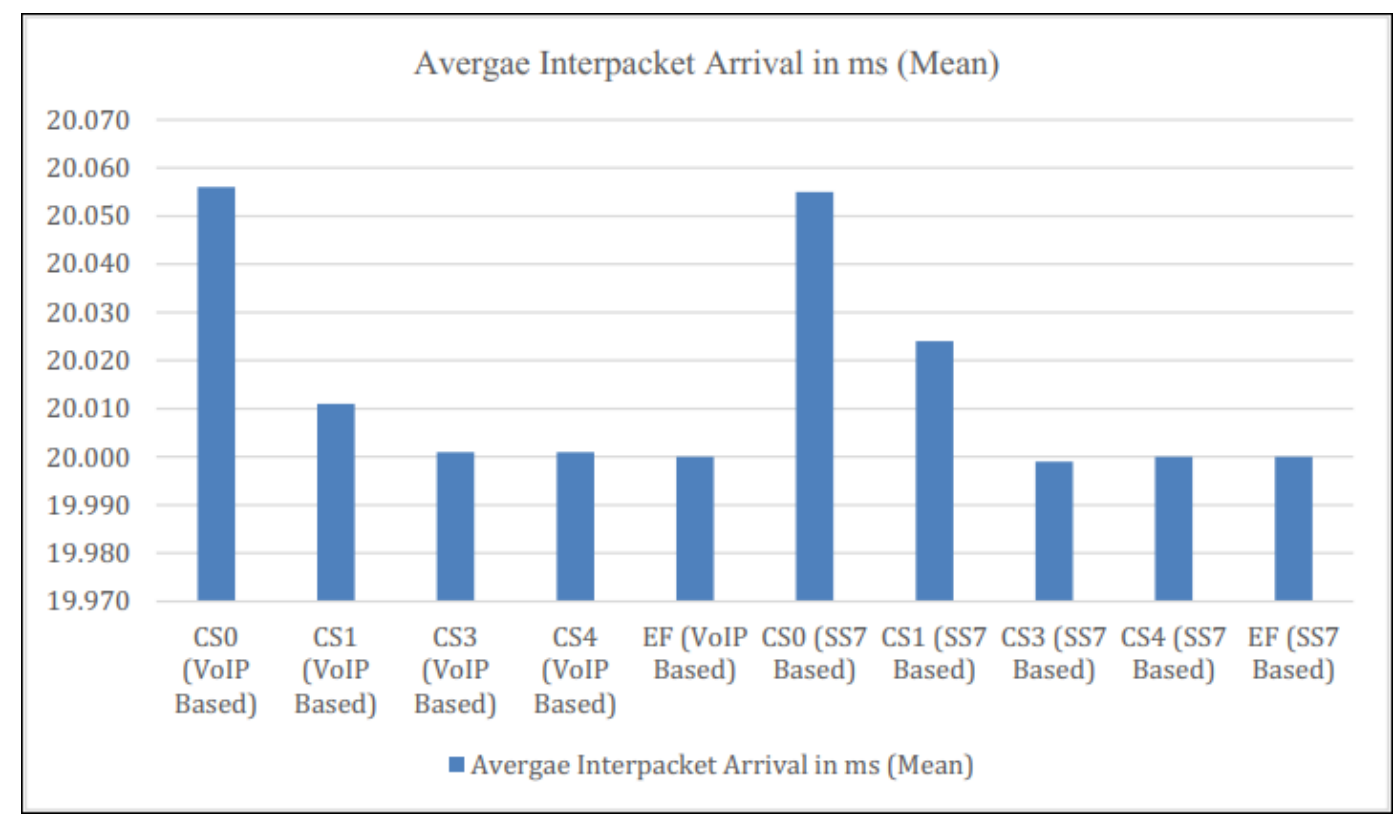

Figure 2. Mean of the average interpacket arrival times in $\mathrm{ms}$

Figure 3 illustrates the jitter's mean values for each of the 10 test cases. All the test cases have a good quality where none of the jitter values exceeded the $7.83 \mathrm{sec}$. The highest mean value for jitter is $3.77 \mathrm{sec}$ that belongs to the CS1 marking of the VoIP-based manual test calls. This value goes hand-in-hand with the VoIP standards of the average jitter where the maximum acceptable jitter's value is $50 \mathrm{~ms}[1,2]$.

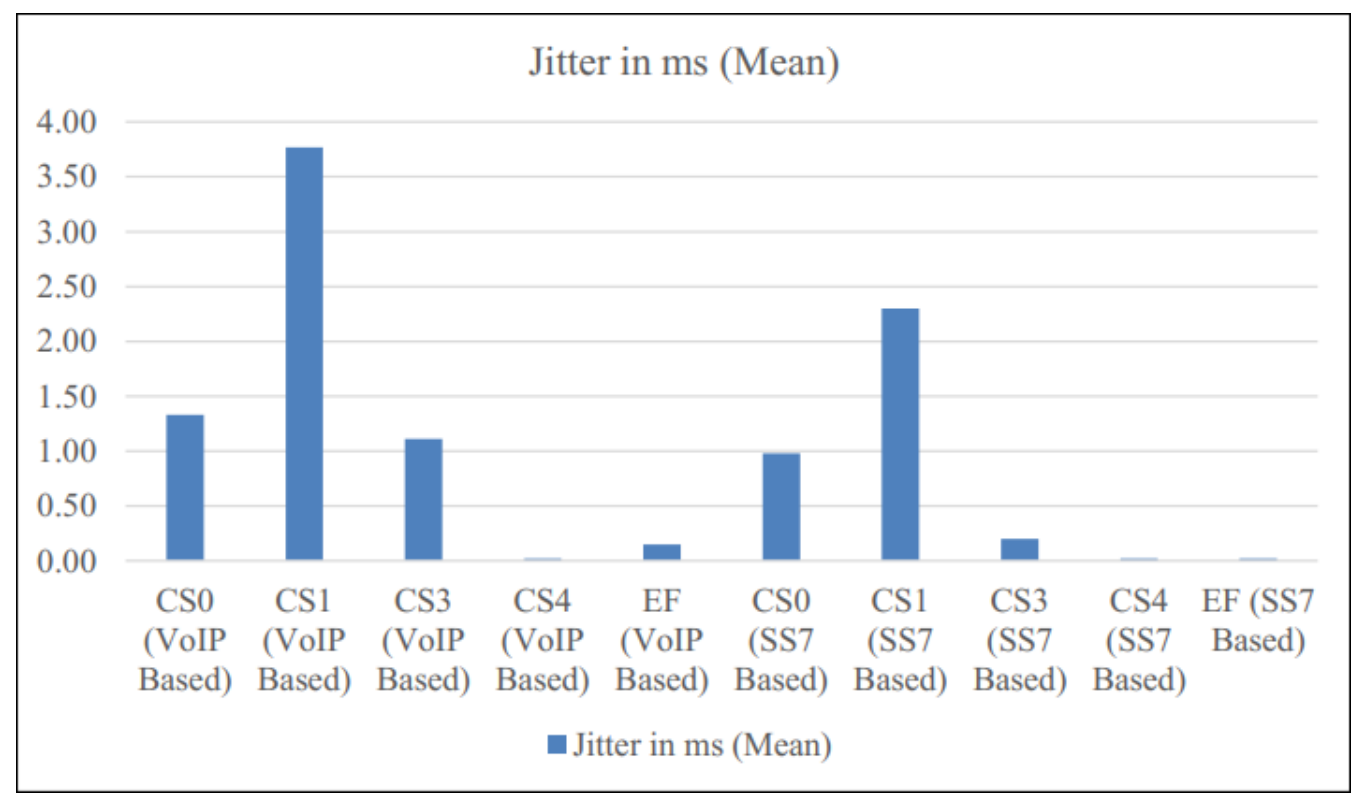

Figure 3. Mean of the jitter in ms 
Figure 4 illustrates the mean values of latencies for each of the 10 test cases. All the test cases have a good quality where none of the latency values exceeded the $54 \mathrm{sec}$. The highest mean value for the latency element is $23.4 \mathrm{sec}$ that belongs to the CSO marking of the VoIP-based manual test calls. This value goes hand-in-hand with the VoIP standards of latency where the maximum acceptable latency's value lies between $80 \mathrm{~ms}$ and $120 \mathrm{~ms}[1,2]$.

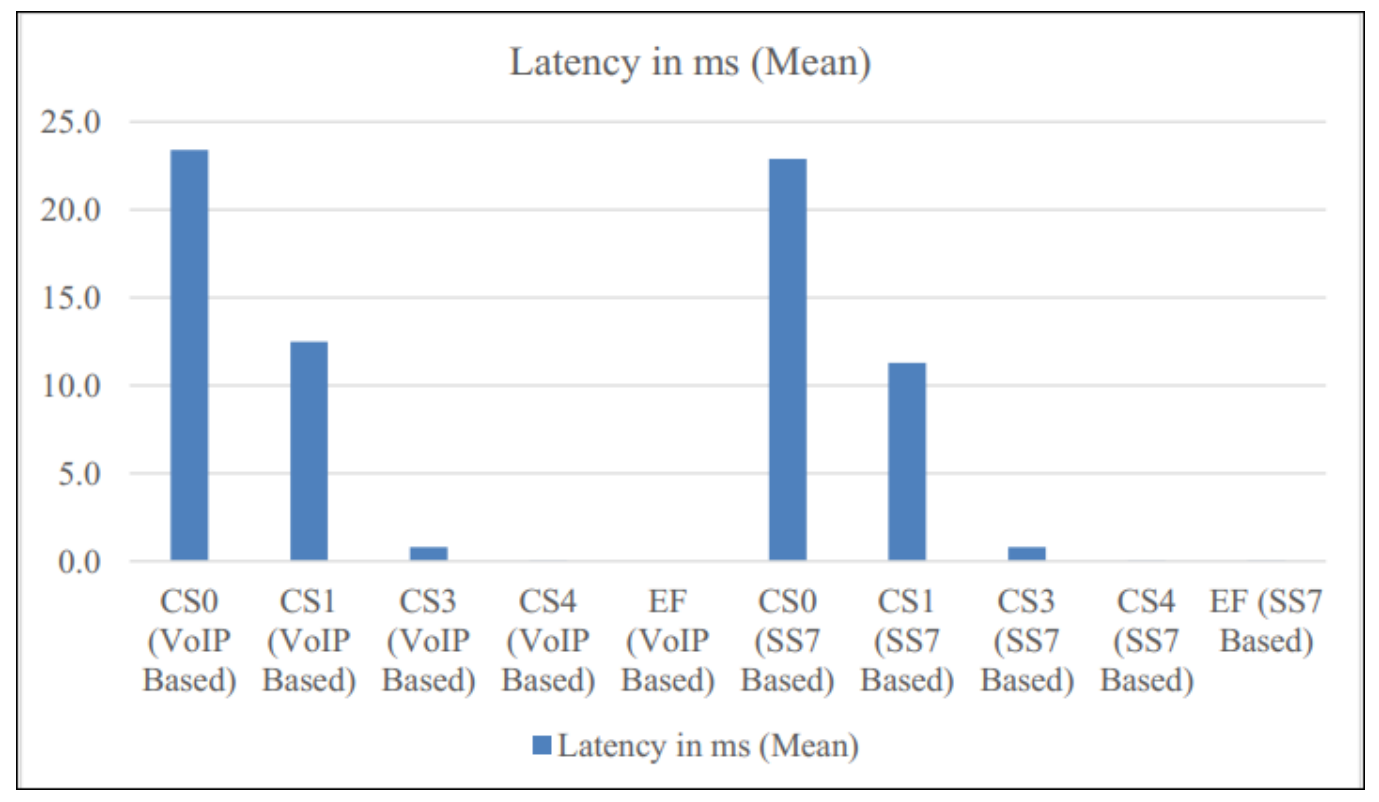

Figure 4. Mean of the latency in ms

We rely on our analysis of the data to answer each of the research questions and the research hypotheses.

- Research Question 1: Will the changes in DSCP markings for the voice traffic have an impact on calls that originate from VoIP networks and terminate in SS7 networks? The examination of the testing data for each of the five VoIP-based test scenarios shows there is some impact from one DSCP marking to another. The impact is minimal that human ear could have not noticed when listening to the phone conversations.

- Research Question 2: Will the changes in DSCP markings for the voice traffic have an impact on calls that originate from SS7 networks and terminate in VoIP networks? The examination of the testing data for each of the five SS7-based test scenarios shows there is some impact from one DSCP marking to another. The impact is minimal that human ear could have not noticed when listening to the phone conversations.

Our research's hypotheses statement states that configuring VoIP traffic with a DSCP marking the value of EF across all the equipment in a broadband HFC network improves VoIP's QoS. VoIP traffic that has a DSCP marking of EF kept its level of good quality and the mixture of video and Internet traffic have no negative impact on VoIP phone calls. When comparing the test data associated with a DSCP marking of EF with the other eight different test cases, the data show a clear difference where VoIP traffic with a DSCP marking of EF improved. None of the test calls associated with the DSCP marking of EF have any packet loss or latency. EF DSCP marking test calls have the same MOS value of 4.40. The interpacket arrival time associated with each of the EF DSCP marking test calls is $20.00[1,2]$. 


\section{CONCLuSions ANd Future Works}

This study found that the higher VoIP traffic's DSCP marking value assignment, the less the chances of higher latency or packet loss in the VoIP traffic. Also, the study found that lower DSCP marking values harm VoIP quality where the probability of running into higher latency or packet loss increases. This study also highlighted that VoIP traffic's DSCP markings are beneficial and have value even when the links between the MGC and the routers are below full utilization level. This research concludes that DSCP markings play an important factor in ensuring that proper prioritization treatment is given to all types of traffic including the VoIP phone services in an IP network when sharing the links with other types of traffic.

Future research should consider studying an additional three of the DSCP markings that include CS2, CS5, and CS6. This experiment tested the five of the DSCP markings while the links between the MGC and the adjacent routers were not at full capacity and future research studies should consider making the same tests but while the links between the MGC and the routers are at full capacity. Other recommendations include making the same tests that were executed in this experiment while changing the DSCP markings either in the adjacent routers or the MGWs. This research did not test any VoIP-based calls to VoIP-based calls within the same network or between two different VoIP networks. Those are some of the options and ideas that future researchers can consider and take into considerations to perform future research studies related to this research, such studies can further strengthen this research and its results $[1,2]$.

\section{REFERENCES}

[1] Daoud, S., \& Qu, Y. (2019). A Comparison Research on DSCP Marking's Impact to the QoS of VoIP-based and SS7-based Phone Calls.

[2] Daoud, S., \& Qu, Y. (2019). Optimizing DSCP Marking to Ensure VoIP's QoS over HFC Network.

[3] Mathiyalakan, S. (2015). VoIP adoption: Issues \& concerns. Communications of the IIMA, 6(2), 3.

[4] Khitmoh, N., Wuttidittachotti, P., \& Daengsi, T. (2014, February). A subjective-VoIP quality estimation model for G. 729 based on native Thai users. In Advanced Communication Technology (ICACT), 2014 16th International Conference on (pp. 48-53). IEEE.

[5] Xiao, Y., Qu, G., \& Kiseon, K. (2015). A new DiffServ edge router with controlled UDP. Chinese Journal of Electronics, 24(1).

[6] Kamarudin, I. E., Sharif, S. A. M., \& Herawan, T. (2013). Performance analysis on the effect of G. 729, Speex and GSM speech codec on $802.11 \mathrm{~g}$ wireless local area network over VoIPvusing packet jitter. International Journal of Control and Automation, 6(4), 387-395.

[7] Broß, J. F., \& Meinel, C. (2008). Can VoIP live up to the QoS standards of traditional wireline telephony? In Telecommunications, 2008. AICT'08. Fourth Advanced International Conference on (pp. 126-132). IEEE.

[8] Singh, P. \& Kaur, R. (2014). VOIP over Wimax: A comprehensive review. International Journal of Computer Science \& Information Technologies, 5(4).

[9] Yuan, Z. (2002). SIP-based VoIP network and its interworking with the PSTN. Electronics \& Communication Engineering Journal, 14(6), 273-282. 
[10] Ali, M. A., Rashid, I., \& Khan, A. A. (2013). Selection of VoIP CODECs for different networks based on QoS analysis. International Journal of Computer Applications (IJCA), 84(5), 14575-2702.

[11] Chen, J. J., Lee, L., \& Tseng, Y. C. (2011). Integrating SIP and IEEE 802.11 e to support handoff and multi-grade QoS for VoIP-over-WLAN applications. Computer Networks, 55(8), 1719-1734.

[12] Assem, H., Malone, D., Dunne, J., \& O'Sullivan, P. (2013, January). Monitoring VoIP call quality using improved simplified E-model. In Computing, Networking and Communications (ICNC), 2013 International Conference on (pp. 927-931). IEEE.

[13] FCC. (2013, June). Local telephone competition: Status as of June30, 2012.

[14] FCC. (2014, June). FCC releases new data on Internet access services and local telephone competition.

[15] Vijayakumar, M., Karthikeyani, V., \& Omar, M. (2013). Implementation of queuing algorithm in multipath dynamic routing architecture for effective and secured data transfer in VoIP. International Journal of Engineering Trends and Technology, 4(4), 1226-1230.

[16] Gray, R. M. (2005). The 1974 origins of VoIP. Signal Processing Magazine, IEEE, 22(4), 87-90.

[17] Vlaovic, B., \& Brezocnik, Z. (2001, July). Packet based telephony. InEUROCON'2001, Trends in Communications, International Conference on. (Vol. 1, pp. 210-213). IEEE.

[18] Bridova, I., Vaculik, M., \& Brida, P. (2011). Impact of background traffic on VoIP QoS parameters in GPON upstream link. Electronics and Electrical Engineering.-Kaunas: Technologija, (1), 104.

[19] Naeem, M., Naz, S., \& Asghar, S. (2013). QoS guarantee for VOIP over wireless LANs. International Journal of Hybrid Information Technology, 6(3), 25-32.

[20] Mohammed, H. A., Ali, A. H., \& Mohammed, H. J. (2013). The affects of different queuing algorithms within the router on QoS VoIP application using OPNET. arXiv preprint arXiv:1302.1642.

[21] Rivas, F. J., Díaz, A., \& Merino, P. (2013). Obtaining more realistic cross-layer QoS measurements: A VoIP over LTE Use Case. Journal of Computer Networks and Communications, 2013.

[22] Mahajan, S., \& Chopra, V. (2013). Performance evaluation of MANET routing protocols with scalability using QoS metrics of VOIP applications. International Journal, 3(2).

[23] Chen, J. J., Lee, L., \& Tseng, Y. C. (2011). Integrating SIP and IEEE 802.11 e to support handoff and multi-grade QoS for VoIP-over-WLAN applications. Computer Networks, 55(8), 1719-1734.

[24] Miraz, M. H., Molvi, S. A., Ali, M., Ganie, M. A., \& Hussein, A. H. (2017). Analysis of QoS of VoIP traffic through WiFi-UMTS networks. arXiv preprint arXiv:1708.05068.

[25] Charonyktakis, P., Plakia, M., Tsamardinos, I., \& Papadopouli, M. (2016). On user-centric modular qoe prediction for voip based on machine-learning algorithms. IEEE Transactions on mobile computing, 15(6), 1443-1456.

[26] Silva, S., Soares, S., Reis, M. J., Neves, F., \& Assuncao, P. A. (2017, July). A dynamic programming algorithm to select optimal high-priority voice segments using Arduino. In IEEE EUROCON 201717th International Conference on Smart Technologies (pp. 271-276). IEEE. 
[27] Baharudin, M. A. B., Quang, T. M., \& Kamioka, E. (2015). Improvement of handover performance based on bio-inspired approach with received signal strength and mean opinion score. Arabian Journal for Science and Engineering, 40(6), 1623-1636.

[28] ITU-T. (1996). Methods for objective and subjective assessment of quality. ITU-T Recommendation, 830.

[29] Al-Sayyed, R., Pattinson, C., \& Dacre, T. (2007, February). VoIP and database traffic coexistence over IEEE 802.11 b WLAN with redundancy. In Proceedings of the International Conference on Computer, Information and Systems Science and Engineering (pp. 25-27).

[30] Vijayakumar, M., Karthikeyani, V., \& Omar, M. (2013). Implementation of queuing algorithm in multipath dynamic routing architecture for effective and secured data transfer in VoIP. International Journal of Engineering Trends and Technology, 4(4), 1226-1230.

[31] Chen, S., Wang, X., \& Jajodia, S. (2006). On the anonymity and traceability of peer-to-peer VoIP calls. IEEE Network, 20(5), 32-37.

[32] Ahmed, D. T., \& Shirmohammadi, S. (2012). Improving online gaming experience using location awareness and interaction details. Multimedia Tools and Applications, 61(1), 163-180.

[33] Englander, M. (2012). The interview: Data collection in descriptive phenomenological human scientific research*. Journal of Phenomenological Psychology, 43(1), 13-35.

[34] Martinek, R., \& Zidek, J. (2012). Application of synthetic instrumentation that applies the trend of software-based approach for measuring on the field of modern wireless transfer systems. International Journal of Digital Information and Wireless Communications (IJDIWC), 2(3), 208-221.

[35] Creswell, J. (2013). Qualitative inquiry and research design: choosing among five approaches. Los Angeles: SAGE Publications.

[36] Raychaudhuri, D., Seskar, I., Ott, M., Ganu, S., Ramachandran, K., Kremo, H., ... \& Singh, M. (2005, March). Overview of the ORBIT radio grid testbed for evaluation of nextgeneration wireless network protocols. In Wireless Communications and Networking Conference, 2005 IEEE (Vol. 3, pp. 16641669). IEEE.

\section{AUTHOR}

Dr. Shaher Daoud currently is a faculty member of the School of Computer Science, Colorado Technical University. Colorado Springs, USA. He also has over 29 years of Telecom industrial working experience.

Dr. Yanzhen Qu currently is a professor of the school of Computer Science, Colorado Technical University, Colorado Springs, USA. He has also worked in the Telecom and Software Development industry for over $20+$ years. 